\title{
3D MODEL GENERATION FROM UAV: HISTORICAL MOSQUE (MASJID LAMA NILAI)
}

\author{
Nadhirah Hani Mohd Nasir \& Khairul Nizam Tahar* \\ Centre of Studies for Surveying Science and Geomatics, Faculty of Architecture, Planning and Surveying, \\ Universiti Teknologi MARA, 40450 Shah Alam, Selangor Darul Ehsan, Malaysia \\ nadhirahhanie@gmail.com; nizamtahar@gmail.com* \\ *Corresponding author
}

KEY WORDS:3D Model, historical mosque, UAV, accuracy, rendering, texturing

\begin{abstract}
:
Preserving cultural heritage and historic sites is an important issue. These sites are subjected to erosion and vandalism, and, as long-lived artifacts, they have gone through many phases of construction, damage and repair. It is important to keep an accurate record of these sites using the 3-D model building technology as they currently are, so that preservationists can track changes, foresee structural problems, and allow a wider audience to "virtually" see and tour these sites. Due to the complexity of these sites, building 3-D models is time consuming and difficult, usually involving much manual effort. This study discusses new methods that can reduce the time to build a model using the Unmanned Aerial Vehicle method. This study aims to develop a 3D model of a historical mosque using UAV photogrammetry. In order to achieve this, the data acquisition set of Masjid Lama Nilai, Negeri Sembilan was captured by using an Unmanned Aerial Vehicle. In addition, accuracy assessment between the actual and measured values is made. Besides that, a comparison between the rendering $3 \mathrm{D}$ model and texturing 3D model is also carried out through this study.
\end{abstract}

\section{INTRODUCTION}

Retaining historical building is an important issue. The development of the latest digital 3D scanning device has created new means to retain these sites digitally and to retain the historic trace by photorealistic 3D models and building geometry. Since the 1930 s, photogrammetry is the science behind the creation of nearly every topographic map (Betram, et al., 2014). Besides that, photogrammetry is also an appropriate instrument to deliver information about vegetation cover and man-made objects (Hassan \& Nawawi, 2014). A 3D model is an object that has height, width and depth like any object in the real world (Singh, et al., 2013). The theory of 3D modelling nowadays is growing in popularity as Points of Interest (POI) in navigation applications such as mobile applications for city travel, digital city guides, city maps in personal mobility devices and vehicle navigation systems (Urey, 2013). There are three main models of 3D models depending upon the different types of shape and technique used for imaging (Clark, et al., 2015). Furthermore, there are two techniques to generate 3D models which are terrestrial close range and close range using Unmanned Aerial Vehicle (UAV).

Aerial photogrammetry and close range photogrammetry projects involve similar processing steps to the 3D model generation (Morgenthaler, et al., 2012). These steps can be characterised as image acquisition, determining the interior and exterior orientation parameters of images, and automatic or manual extraction of geometric features of interest stages. Close range photogrammetry (CRP) is a photograph captured at short distances with higher Ground Sample Distance (GSD). Close-range is also known as CRP or terrestrial in which it has an object-to-camera distance of less than $300 \mathrm{~m}$. Theoretically, there is no limit to the resolution that can be achieved since a variation of cameras and platforms may be used to obtain the photographic images for CRP processing. Meanwhile, aerial photogrammetry uses ground coordinate information and large-format imagery to successfully reconstruct the geometry of a part of the earth in an effective environment. In this effective environment, vertical measurements and reliable horizontal can be recorded and made or compiled directly into a geospatial data file. In the current scenario, there are three main methods used to construct a virtual 3D model. The first is the sketch method which is simple and does not fulfil any engineering requirement. Second is the procedural method which is not easy for amateur users and the third is the CRP method which is not fitting for large landscape modelling (Singh, et al., 2013).

The rendering model is very smooth, shows realistic 3D model in $2.5 \mathrm{D}$ and $2 \mathrm{D}$ image, brings large enhancements in efficiency of production, clarity and realism in finished image especially for 3D rendering, can be completed from autocad application, hand drawing or evenly scanned hard copies and mostly used in 3D graphics and CAD (Niethammer, et al., 2010). The texturing model shows real surface, realistic 3D model in 3D image and mostly used in building, medical industry in detailed models of organs.

This study proposes UAV photogrammetry as a tool to capture the composition of a historical mosque and generate a 3D model of the mosque. UAV photogrammetry defines photogrammetric as measurement platforms which operates either semi-autonomously, autonomously, or remotely controlled without an on-board pilot. UAV photogrammetry is known as anew photogrammetric measurement instrument (Eisenbeiss, 2006; Perez, et al., 2013). UAV photogrammetry not only opens many new applications in combining aerial, terrestrial photogrammetry and close range field, but also introduces low-cost alternatives to the traditional manned aerial photogrammetry (Jain, et al., 2014). Besides that, UAV can also accumulate high quality aerial optical images for city modelling using photogrammetric techniques (Gruen, et al., 2013). There are three main categories of UAVs which are strategically UAV, special tasks UAV and tactical UAV (McGovern, 2014). Tactical UAV includes mini, micro, close, short, medium-range endurance, medium-range, medium altitude long endurance systems, low altitude deep penetration, and low altitude long endurance with flight altitude from a few hundred meters to $5 \mathrm{~km}$ 
and the endurance from some minutes to 2-3 days (Rinaudo, et al., 2012). Strategically UAV includes stratospheric, exo-stratospheric systems and high altitude long endurance which can fly higher than $20,000 \mathrm{~m}$ altitude and have an endurance of 2-4 days. Special tasks are for example unmanned flight autonomous vehicles, dangerous and decoy systems (Tahar, et al., 2014). Therefore, UAV photogrammetry is an important source of image data for general $3 \mathrm{D}$ reconstruction purposes of man-made structures.

\section{METHODOLOGY}

The methodology of this study can be divided into four phases which are referred to as phase I, phase II, phase III and phase IV. Phase I is an initial exploration of issues known as the preliminary study. Phase II is data acquisition for two processes which are flight planning and camera calibration. Phase III is data processing for generating a 3D model using five steps which are image masking, image align, image dense cloud, image mesh and image texture. Phase IV is the results and analysis obtained from the 3D model which are assessments of the accuracy and rendering of the 3D model. Figure 1 shows the research methodology of this study.

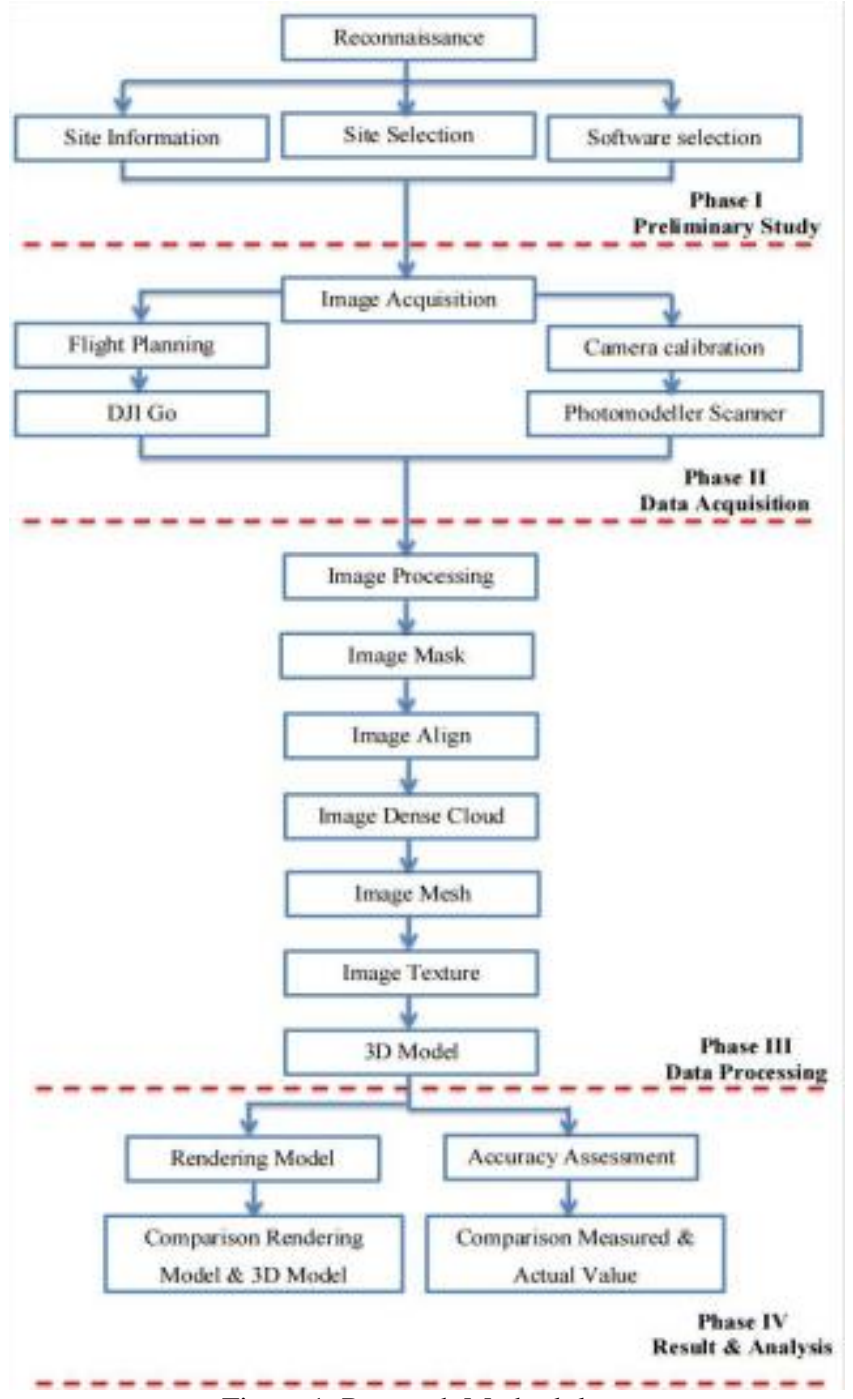

Figure 1. Research Methodology

\subsection{Location of Study}

Selection of the study area was in Negeri Sembilan which is the state on the southwest coast of Peninsular Malaysia, south of capital Kuala Lumpur, with Pahang in the east, Malacca and Johor to the south and bordering Selangor on the north. It is a historical mosque in Nilai, Negeri Sembilan, Malaysia. Masjid Lama Nilai is also known as Masjid Kariah Pekan Nilai and it was built in 1928. It was founded and built by the locals. The mosque site was given by the state government. The building of the mosque was repaired and upgraded in the year 1995 and the latest in 2015.

\subsection{Image Acquisition}

The data for the 3D model was collected using the Phantom 3 Professional. Before starting the work, camera calibration was carried out first. This is to ensure that the instrument is in good condition. The software used to process the result of camera calibration was the photomodeller software. DJI GO is the flight planning used in this study to plan the flight throughout this study. Multiple overlapping photos with 80 to $90 \%$ overlap model were taken using autonomous programmed flight path.

\subsection{Camera Calibration}

Camera calibration is needed to identify the elements of interior orientation such as focal length, lens distortion, principle point, and resolution of lens. This is useful to check whether the instrument can be used and solve internal camera parameters. The criteria to pass the camera calibration results are:1) the point's photo coverage must be at least $80 \%$ and, 2) the residual of the photos should be less than 1 pixel. Table 1 shows the results of camera calibration from seven sets of calibration.

\begin{tabular}{|c|c|c|c|c|c|c|c|c|}
\hline & Fimat) & $X_{p}$ (iman) & $V_{p}(\operatorname{ma})$ & $F_{n}(\operatorname{man})$ & $\mathbf{E}(\mathbf{m} \mathbf{m})$ & KI 1 (ma) & $\mathrm{K} 2(\mathrm{ma})$ & $\begin{array}{l}\text { Couress } \\
\text { Perentag }\end{array}$ \\
\hline Sert & 3767 & 8230 & 2.432 & 6.543 & 4.874 & $634+094$ & $4,343 e-066$ & 725 \\
\hline Set 2 & 2764 & 3221 & 2.435 & 0.496 & 4.63 & cosesest & tosstease & Tos \\
\hline Set 3 & 2.70 & 3720 & 2.234 & 0.901 & 4.57 & T.45:-004 & $-1.3340-065$ & S4: \\
\hline Set 4 & $3, \pi$ & $3: 2 \times 3$ & 2,436 & 6.317 & 4.575 & A.35ste004 & $-3.5450-064$ & Tys: \\
\hline Seets & 270 & 1238 & 2.49 & s.4s & 45 & $1 \cos 2 n-000$ & s.sikenes & 785, \\
\hline Sete & $2 \pi 2$ & 2208 & 2.39 & 2.69 & 403 & $1053 e-003$ & s.askeves & 786 \\
\hline bet? & 3780 & 3269 & 2,489 & 6.590 & 4.173 & 1. Alseves & .5.35rewes & hes. \\
\hline
\end{tabular}

Based on Table 1, it shows that the internal geometry of UAV's camera can be defined using the appropriate technique. The percentage of coverage can be achieved more than $70 \%$. The focal length values for all data sets is not quite different and not significant. The others parameters also show the value close to each other.

\subsection{Flight Planning}

The specific autonomous software is used to create waypoints which also represents the pre-planning route for this mission. Overlapping photos of an object by 80 to $90 \%$ would be impossible to be completed accurately by pilot navigation. In this study, the images were taken every five seconds of lap surrounding the mosque. Three layers of rotation (different altitudes) were taken to assure that every detail of the building was captured carefully by 
DJI GO apps. The drone followed the designed path to capture the whole building of Masjid Lama Nilai. The obstacles to capture the image such as trees, other buildings, electricity poles, lamps and antennas was overcome by making a few orbital paths with different perspectives around the façade of Masjid Lama Nilai. The drone's camera was set to be taken as a JPEG image with ultra-highresolution and high frame rate. Higher resolution will give better results. Figure 2 shows the image captured using the Phantom 3 Pro from different perspectives.

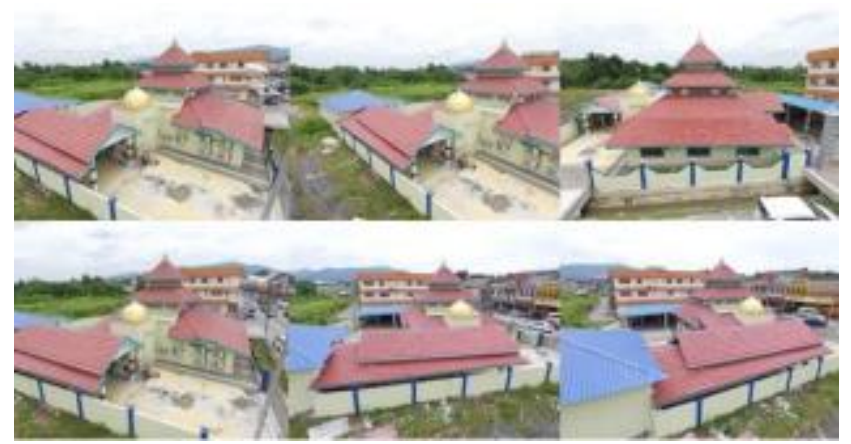

Figure 2. Images capture using Phantom 3 Pro from different perspective

\section{RESULTS AND ANALYSIS}

The final 3D model generated with the Agisoft software had a textured realistic look from any viewing angles. The 3D model results were divided into two categories which were accuracy assessment and rendering the model.

\subsection{Accuracy Assessment}

The three dimensional structure of the building is measured by a conventional method and translated into a model using the scaling method. Scaling method is used to predefine the model based on the actual measurement. Based on the actual measurement as an input, the scaling method allow the software to transform the generated model to the actual scale model. Thus, the output accuracy of the model is much better and nearer to the actual value. In this case, all distances were measured using a tape measurement. Therefore, the distances were compared by actual value and measured value. There is a difference in the actual value and measured value. There are 31 point marks places in this model as shown in Figure 3. Table 2 shows the actual value of the 3D model and the comparison of actual and measured values. The RMSE of the actual and measured values is $\pm 0.05 \mathrm{~m}$

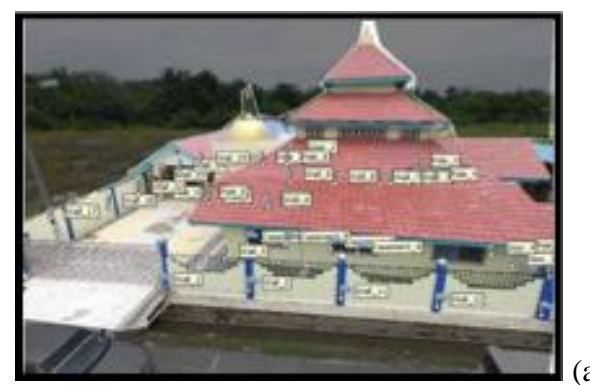

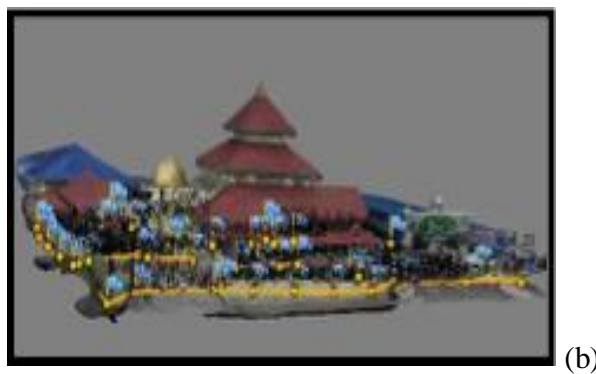

Figure 3. Mark point using Agisoft software; a) images, b) 3D Model

Table 2. Comparison Actual and Measured Value

\begin{tabular}{|c|c|c|c|c|}
\hline No. & $\begin{array}{c}\text { Building } \\
\text { details }\end{array}$ & $\begin{array}{c}\text { Actual } \\
\text { value } \\
(\mathbf{m})\end{array}$ & $\begin{array}{c}\text { Measured } \\
\text { value (m) }\end{array}$ & $\begin{array}{c}\text { Residuals } \\
(\mathbf{m})\end{array}$ \\
\hline $\mathbf{1}$ & Casement 1 & 1.39 & 1.39 & 0.00 \\
\hline $\mathbf{2}$ & Casement 2 & 1.39 & 1.39 & 0.00 \\
\hline $\mathbf{3}$ & Door 1 & 0.90 & 0.90 & 0.00 \\
\hline $\mathbf{4}$ & Door 2 & 0.90 & 0.90 & 0.00 \\
\hline $\mathbf{5}$ & Front gate & 5.13 & 5.10 & 0.03 \\
\hline $\mathbf{6}$ & Wall 1 & 4.00 & 4.00 & 0.00 \\
\hline $\mathbf{7}$ & Pole 1 & 1.70 & 1.70 & 0.00 \\
\hline $\mathbf{8}$ & Pole 2 & 5.55 & 5.50 & 0.05 \\
\hline $\mathbf{9}$ & Pole 3 & 3.10 & 3.00 & 0.10 \\
\hline $\mathbf{1 0}$ & Pole 4 & 3.00 & 3.00 & 0.00 \\
\hline $\mathbf{1 1}$ & Back stair & 0.80 & 0.90 & -0.10 \\
\hline $\mathbf{1 2}$ & Front stair & 1.26 & 1.25 & 0.01 \\
\hline $\mathbf{1 3}$ & Wall 2 & 2.65 & 2.70 & -0.05 \\
\hline $\mathbf{1 4}$ & Back gate & 4.65 & 4.60 & 0.05 \\
\hline $\mathbf{1 5}$ & Wall 3 & 2.75 & 2.70 & 0.05 \\
\hline $\mathbf{1 6}$ & Wall 4 & 3.18 & 3.00 & 0.18 \\
\hline $\mathbf{1 7}$ & Wall 5 & 6.00 & 6.00 & 0.00 \\
\hline $\mathbf{1 8}$ & Wall 6 & 3.00 & 3.00 & 0.00 \\
\hline $\mathbf{1 9}$ & Wall 7 & 3.00 & 3.00 & 0.00 \\
\hline $\mathbf{2 0}$ & Wall 8 & 1.56 & 1.55 & 0.01 \\
\hline $\mathbf{2 1}$ & Wall 9 & 2.75 & 2.75 & 0.00 \\
\hline $\mathbf{2 2}$ & Wall 10 & 2.75 & 2.75 & 0.00 \\
\hline $\mathbf{2 3}$ & Wall 11 & 1.55 & 1.55 & 0.00 \\
\hline $\mathbf{2 4}$ & Wall 12 & 3.55 & 3.55 & 0.00 \\
\hline $\mathbf{2 5}$ & Wall 13 & 3.00 & 3.00 & 0.00 \\
\hline $\mathbf{2 6}$ & Wall 14 & 3.00 & 3.00 & 0.00 \\
\hline $\mathbf{2 7}$ & Wall 15 & 5.25 & 5.20 & 0.05 \\
\hline $\mathbf{2 8}$ & Wall 16 & 10.95 & 11.00 & -0.05 \\
\hline $\mathbf{2 9}$ & Wall 17 & 3.00 & 3.00 & 0.00 \\
\hline $\mathbf{3 0}$ & Wall 18 & 3.00 & 3.00 & 0.00 \\
\hline $\mathbf{3 1}$ & Wall 19 & 3.00 & 3.00 & 0.00 \\
\hline & & & & \\
\hline
\end{tabular}

Table 2 indicates the $60 \%$ of the residuals gave zero value. It is means that the 3D model has the same value with the actual measurement. While the other sample has the residual at the centimetre and millimetre level.

\subsection{Rendering 3D Model}

$3 \mathrm{D}$ rendering is the $3 \mathrm{D}$ computer graphics process. It is an automatic process to convert a $3 \mathrm{D}$ wireframe model into $2 \mathrm{D}$ images with 3D photorealistic effects. Rendering gives a final 
photorealistic look for a 3D model. In this study, a complete 3D model of a mosque was created and the texture of the rendering model is smoother than the $3 \mathrm{D}$ model. Figure 4 shows the result after rendering and texturing the 3D model.

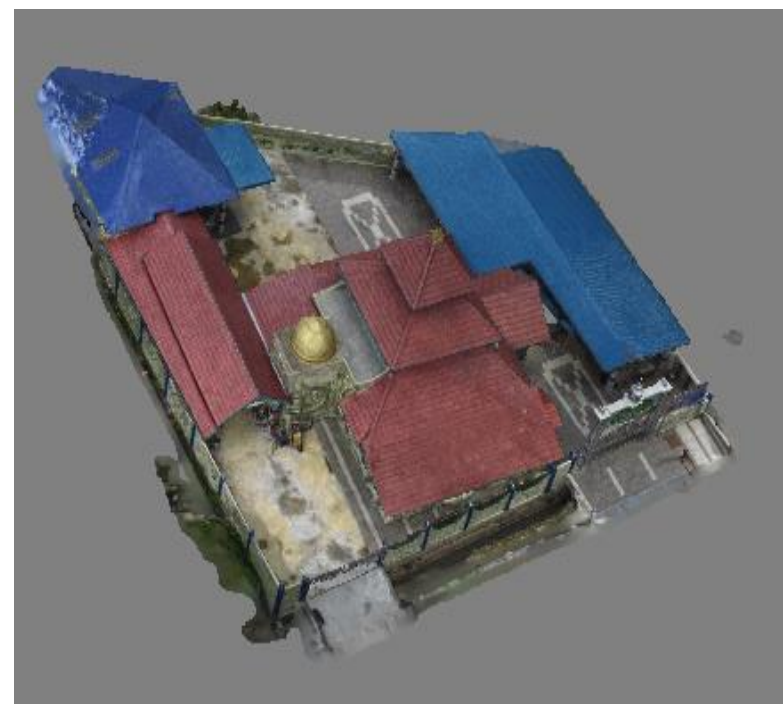

Figure 4. Results of 3D Model after rendering and texturing

\section{CONCLUSIONS AND RECOMMENDATIONS}

This study has successfully achieved its first objective which is to generate a 3D model of a historical mosque using UAV images. Image taken using UAV is a very cost effective solution for image data acquisition of 3D modelling of the mosque. The image processing involved the image captured around the mosque. The products are textured, photorealistic and scalable 3D models of the mosque. Thus, the methodology which included image masking, image align, image dense cloud, image mesh and image texture used to create a 3D model of the mosque using UAV is completed. This study assessed the accuracy of the $3 \mathrm{D}$ model to ensure the generated 3D model is near to the object. Scaling method is used by entering the measured dimensional value which is the actual and measured values.

The accuracy of the model is based on the input value. In this study, $3 \mathrm{D}$ model of mosque's accuracy by the root mean squared error is $\pm 0.05 \mathrm{~m}$. The method used to assess the accuracy of the $3 \mathrm{D}$ model is by comparing the actual and measured values. Besides that, the textured quality of a model is dependent on camera resolution. Most of the steps used in this method are automatic. Hence, this photogrammetric method is much better than the conventional method and it is also time saving because this method has more advantages than any previous method available for 3D modelling of the mosque. The aim of this study is to develop a 3D model of a historical mosque using UAV photogrammetry. 3D models of any historical mosque or other buildings are also acceptable to act as a prototype of the 3D model. The method used in this study which generates a 3D model using UAV is also suitable and useful for various kinds of applications that involve 3D model applications such as $3 \mathrm{D}$ navigation, 3D city model and 3D game industry. It is recommended that future researchers study the effects of 3D model from different viewing angles. The distance between UAV and object can also be investigated in order to obtain high accuracy results. In future, the flight condition such as height of UAV over ground, distance to objects, camera parameters and resolution can be considered to improve the accuracy of 3D model.

\section{ACKNOWLEDGEMENTS}

Faculty of Architecture, Planning, and Surveying UniversitiTeknologi MARA (UiTM), Research Management Institute (RMi) and Ministry of Higher Education (MOHE) are greatly acknowledged for providing the fund BESTARI 600IRMI/MyRA 5/3/BESTARI $(001 / 2017)$ to enable this research to be carried out. The authors would also like to thank the people who were directly or indirectly involved in this research.

\section{REFERENCES}

Betram, T. T., Bock, T. T., Bulgakov, A. G., \&Evgenov, A. A. (2014). Generation the 3D Model Building by Using the Quadcopter. The 31st International Symposium on Automation and Robotics in Construction and Mining.

Clark, R. A., Punzo, G., Dobie, G., Macleod, C. N., Summan, R., Pierce, G. (2015). 3D Model Generation using an Airborne Swarm. 41ST Annual Review Of Progress In Quantitative Nondestructive. Boise, Idaho: AIP Conference Proceedings.

Eisenbeiss, H., \& Zhand, L. (2006). Comparison of DSMs generated from mini UAV imagery and terrestrial laserscanner in a cultural heritage application. The International Archives of Photogrammetry, Remote Sensing and Spatial Information Sciences, Vol. XXXVI part 5.

Gruen, A., Huang, X., Qin, R., Du, T., Fang, W., Boavida, J. (2013). Joint Processing of UAV Imagery and Terrestrial Mobile Mapping System Data for Very High Resolution City Modeling. International Archives of the Photogrammetry, Remote Sensing and Spatial Information Sciences, 175-182.

Hassan, A. S., \& Nawawi, M. S. (2014). Malay Architectural Heritage on Timber Construction Technique of the Traditional KampungLaut Old Mosque, Malaysia .Asian Social Science.

Jain, K., Mandla, R. V., \& Singh, S. P. (2014). A New Approach Towards Image Based Virtual 3D City Modelling by Using Close Range Photogrammetry. ISPRS Annals of the Photogrammetry, Remote Sensing and Spatial Information Sciences.

McGovern, E. (2014). Ubiquitous UAVs. Society of Chartered Surveyors.

Morgenthaler, S., Braun, T., Zhao, Z., Staub, T., \& Anwander, M. (2012). A Mobile Wireless Mesh Network Using Unmanned Aerial Vehicles. Swiss National Science Foundation.

Niethammer, U., Rothmund, S., James, M. R., Traveletti, J., \& Joswig, M. (2010). UAV-based remote sensing of landslides. Int. Archives of Photogrammetry, Remote Sensing and Spatial Information Sciences.

Perez, M., Aguera, F., \& Carvajal, F. (2013). Low Cost Surveying Using an Unmanned Aerial Vehicle. International Archives of the Photogrammetry, Remote Sensing and Spatial Information 
The International Archives of the Photogrammetry, Remote Sensing and Spatial Information Sciences, Volume XLII-2/W6, 2017 International Conference on Unmanned Aerial Vehicles in Geomatics, 4-7 September 2017, Bonn, Germany

Sciences.

Rinaudo, F., Chiabrando, F., Lingus, A., \& Spano, A. (2012). Archaeological site monitoring: UAV photogrammetry could be an answer. Int. Archives of Photogrammetry, Remote Sensing and Spatial Information Sciences.

Singh, S. P., Jain, K., \&Mandla, R. V. (2013). Virtual 3D Campus Modeling by using Close Range Photogrammetry.American
Journal of Civil Engineering and Architecture, 200- 205.

Tahar, K.N. Akib, W. W., Ahmad, A.,\&Mohd, W. W. (2014). A generic approach for photogrammetric survey using a sixrotor. IOP Conf. Series: Earth and Environmental Science.

Urey, O. (2013). Transformation of Minarets in Contemporary Mosque Architecture in Turkey. International Journal of Science Culture and Sport. 